\title{
An Adaptive Approach to Enhanced Traffic Signal Optimization by using Soft-computing Techniques
}

\author{
Eusebio Angulo $^{\mathrm{a}}$, Francisco P. Romero ${ }^{\mathrm{b}}$, Ricardo García ${ }^{\mathrm{a}}$, \\ Jesús Serrano-Guerrero ${ }^{a}$, José A. Olivas ${ }^{\mathrm{a}}$ \\ ${ }^{a}$ Escuela Superior de Informática, Universidad de Castilla - La Mancha, \\ Paseo de la Universidad, 4, 13071, Ciudad Real, Spain \\ ${ }^{b}$ Escuela Universitaria de Ingeniería Técnica Industrial, Universidad de Castilla - La Mancha \\ Avda. Carlos III, s/n, 45071, Toledo, Spain
}

\begin{abstract}
This paper presents an application of diverse soft-computing techniques to adaptive traffic light controls. The proposed methodology consists of two main phases: off-line and on-line. First, clustering techniques and optimization methods are used at the off-line stage to discover the prototypes which characterize the traffic mobility patterns at an intersection. After this process an optimum timing plan is decided for each mobility pattern detected. In the on-line phase, a prediction model is then constructed on the basis of the prototypes found. Fuzzy Logic based techniques are used to formally represent the prototypes in the prediction model and these prototypes are parametrically defined through frameworks. During the on-line phase an intelligent transportation system, by using the prediction model, matches the current traffic conditions to the mobility patterns detected at the off-line stage in order to identify the most suitable one to be used. The use of these techniques supposes a substantial contribution to the significance of the prediction model, making it robust in the face of anomalous mobility patterns, and efficient from the point of view of real-time computation.
\end{abstract}

Keywords: Traffic signal control; Clustering; Soft-computing; Fuzzy Logic; Intelligent transportation system;

\section{Introduction}

The continued development of infrastructures in all cities implies problems related to transportation systems such as noise, pollution or traffic jams that affect a large number of people. However new technologies provide new tools useful for partially solving these problems. Especially, traffic monitoring technologies can help to control the traffic lights, i.e., the time required for a transition between the red and green signals of a traffic signal; and hence the effects of traffic noise or jams can be reduced.

In order to solve some of the previously mentioned problems it is necessary to design an optimum signal timing plan. The signal timing plans seek to optimize i) the cycle length of a signal, that is defined as the duration time from the center of the red phase to the center

\footnotetext{
${ }^{\sqrt{2}}$ Fax: (+34) 926295354, Tel.: (+34) $926295300+\operatorname{Ext}(6485)$

E-mail: \{Eusebio.Angulo, FranciscoP.Romero, Ricardo.Garcia, Jesus.Serrano, Joseangel.olivas \} @uclm.es
} Preprint submitted to Expert Systems with Applications of the next red phase ii) green splits, the percentage of time devoted to each phase during a cycle and iii) offsets, the phase difference between signal transitions at consecutive intersections regulated by traffic signals.

Next, a taxonomy of strategies that can be applied to the traffic signal control can be seen according to the representation of the real traffic network: non-adaptive traffic signal control approaches and adaptive traffic signal control approaches.

The non-adaptive signal control approaches. Represent the transportation system by means of macroscopic models or microscopic traffic simulators. These methods allow different actions within the traffic system to be assessed but they do not use data on existing traffic conditions when the traffic signal systems are activated.

They can be classified according to two temporal planning contexts: static methods and dynamic methods.

The static methods assume a stationary situation of the traffic is assumed, and their objective is to obtain the signal regulation of a set of intersections within the 
network. This problem has been formulated through a Mathematical Program with Equilibrium Constraints (MPEC). The results of these models are signal regulations with fixed times for each cycle.

A fairly large volume of research has been focused on these methods for optimizing timing plans. Most of the works use techniques such as genetic algorithms, queue theory, dynamic programming, neural networks, etc. A detailed discussion of these methods is beyond the scope of this paper but the following works Van Leeuwaarden (2006), Rouphail et al. (2000), López et al. (1999), Spall and Chin (1994), Sánchez et al. (2008), Dong et al. (2005) and Sánchez et al. (2004) can be seen as a representative sample of the application of these techniques.

The dynamic methods consider the non-stationarity demand patterns. These methods divide one day into intervals, known as Time-Of-Day (TOD) intervals, and it is assumed that for each TOD interval the traffic flow is roughly stationary. Relatively little attention has been devoted to these methods. Papers to address this matter Wong and Woon (2006) and Wong and Woon (2008) present a novel method for optimizing traffic timing plans via the use of clustering algorithms and optimization methods to generate TOD intervals automatically. Other techniques such as genetic algorithms Park et al. (2003) have been used in order to deal with this problem.

The appearance of new traffic monitoring technologies permits the real-time availability of precise data with regard to the traffic conditions and makes possible the adaptive signal control approaches. These methods adjust a real-time timing plan to adapt to the changes of traffic flow.

Following these ideas, Ibrahim et al. (2005) and Ibrahim et al. (2007) created a software tool for traffic light regulation by using wireless propagation models.

Traffic patterns present variations during the entire day, depending on the day of the week, the month of the year, etc. These variations could be predictable and included in a suitable set of TOD intervals. But there are many other circumstances which are not predictable such as sporting or cultural events, demonstrations, roadworks, etc., that can only be detected by an intelligent real-time system. This challenge provides a clear motivation for the creation of adaptive methods for controlling traffic signals.

Asar et al. (2008) proposes an Artificial Neural Network (ANN) as the basis of a traffic signal controller. The Particle Swarm Optimization method has been used for the learning rule of ANN and the optimization of timing plans. In Ma et al. (2008) a traffic signal con- troller for intersections is designed based on Fuzzy Logic. Lee et al. (2005) presents a research that is focused on real-time adaptive signal optimization using genetic algorithms. In this paper, the test results indicate that the real-time genetic control outperformed fixedsignal timing plan in all scenarios. Shelby (2004) identifies deficiencies in the current generation of adaptivecontrol algorithms.

New technologies allow monitoring of many features that could be integrated into the control systems. For instance, Liu et al. (2002) measures the number of vehicles that are queuing at a traffic control.

Stoilova and Stoilov (1998) poses the idea of creating a closed loop system in which the time of the traffic light cycles is calculated in accordance with the acoustic contamination level. According to this idea, a simulation is carried out using only the acoustic contamination level as system input. These simulations obtained better results than those strategies based on traffic lights which have fixed time cycles.

Real-time computational capabilities are identified as the main roadblock to overcome it. This is one of the main motivations of the methodology here proposed. This paper proposes a methodology for controlling signal controlled intersections adaptively. The use of realtime traffic data is essential to tackle this problem.

The use of several soft-computing techniques, such as Fuzzy Logic and Clustering, allow us to obtain more comprehensible and useful results in order to optimize adaptively the traffic timing plans. This approach has been successfully used in other domains such as forest fire prediction Olivas and Romero (2000).

On the other hand, during the on-line phase, one of the previously mentioned plans will be selected by means of the estimation of the traffic conditions at that moment. This fact shows that the proposed methodology can be scalable because the computational load is performed during the off-line phase.

The remainder of the work is organized as follows: Section 2 describes the different tasks that have been carried out to design the mobility patterns-based model. To assess the methodology here proposed, a study case has been developed and explained in detail in Section 3. Finally, some conclusions and future work will be pointed out.

\section{Proposed methodology}

Traffic monitoring technologies allow measuring the real-time traffic conditions. The proposed methodology is based on the presence of this kind of devices in the 


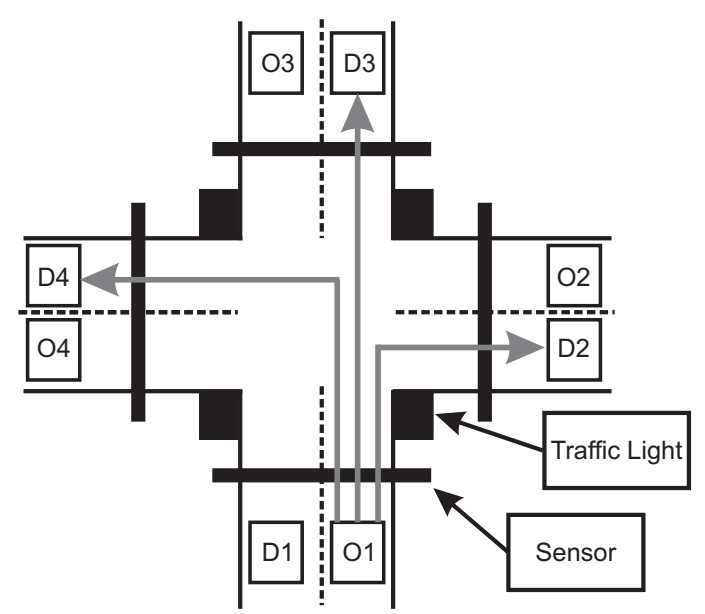

Figure 1: Four lane intersection with no U-turns

transportation systems. For simplicity, only an intersection as shown in Figure 1 is going to be considered. The intersection has sensors for all lanes in order to detect traffic link counts for each time interval.

Figure 1 shows that users who are in origin lane $O 1$ can go to destinations $D 2, D 3$, or $D 4$. There are twelve possible movements at the intersection, of the form $\left(O_{i}, D_{j}\right)$ with $i \neq j ; i, j \in\{1,2,3,4\}$. This intersection has a system of signal control that could be based on our methodology.

The methodology here proposed consists of two different phases: off-line and on-line. The aim of the offline phase is to generate a set of signal timing plans. This timing plan could be applied to regulate the traffic at the intersection according to a set of traffic patterns previously detected by means of a clustering algorithm. Once this phase has been performed, during the on-line phase the current traffic flow is detected using the abovementioned sensors and the current flow and the traffic patterns previously detected by the off-line phase are compared in order to choose the pattern which is able to describe in a precise way the current state of the traffic at the intersection. After choosing the most suitable pattern, the associated optimal signal timing plan is executed to control the traffic in a efficient way. This procedure presents an adaptive mechanism for regulating the behaviour of the different traffic controls that are placed at the intersection depending on different conditions that can take place there.

Figure 2 shows a scheme of the proposed methodology and its different phases. Next, both phases will be described in detail.

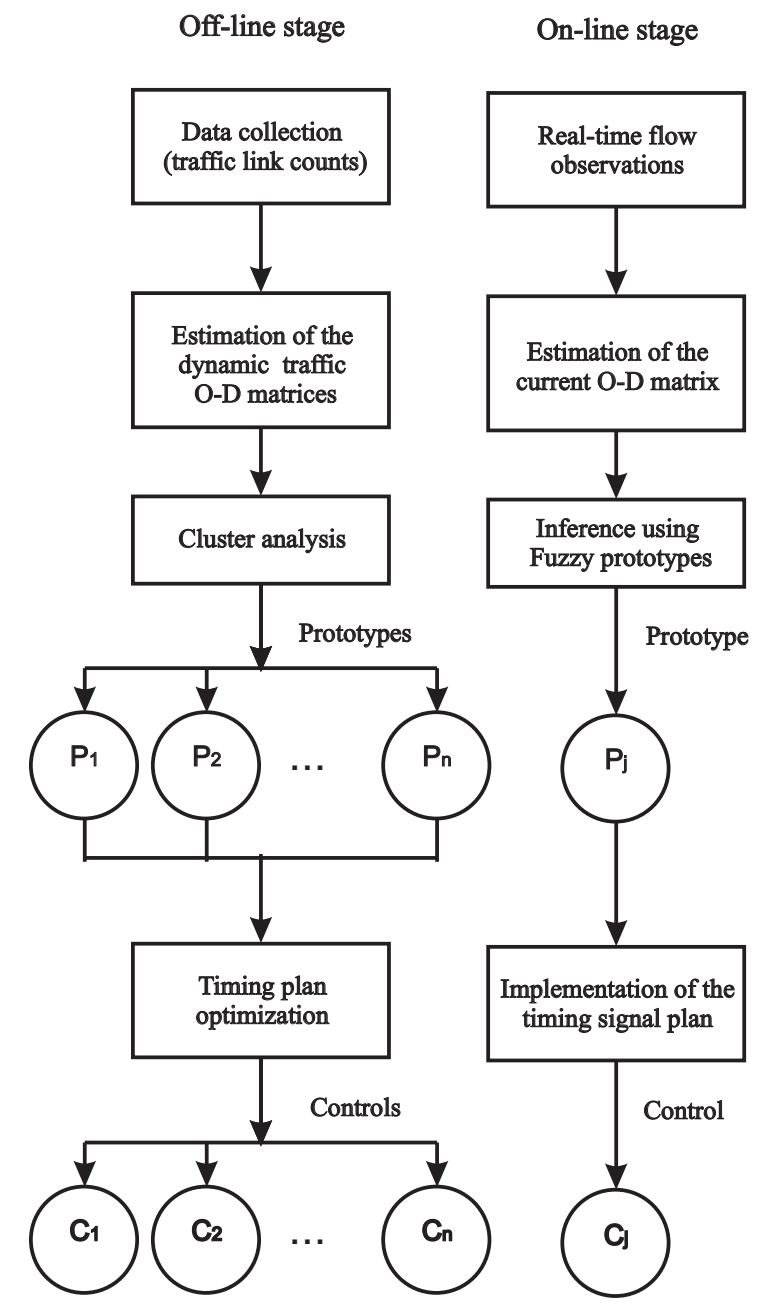

Figure 2: Adaptive Methodology 


\subsection{Off-line phase}

This phase consists of four steps:

1. Data collection. The purpose of this first step of the procedure is to generate a set of observed traffic link counts. For this purpose, a set of days (workdays, weekends, holidays, etc.) has been selected that is able to reflect several possible situations that can happen at the intersection. For each day, a set of traffic link counts is collected at the intersection by using sensors.

2. Estimation of the dynamic traffic origindestination matrices. The origin-destination matrices (O-D matrix from now on) is defined as being the matrix which contains, in the $\left(O_{i}, D_{j}\right)$ cell, the flow (number of vehicles per time unit) which is incorporated into the lane $i$ of the intersection, and which leaves the intersection via lane $j$ at interval time $t$. Let this value be $g_{i j}(t)$ and the O-D matrix by $g(t)$ be defined by $\left(\ldots, g_{i j}(t), \ldots\right)$. It is assumed that U-turn movements are not allowed, i.e., the main diagonal entries $\left(O_{i}, D_{i}\right)$ of the O-D matrix are all zero, i.e. $g_{i i}(t)=0$.

In this phase an O-D matrix is computed for each time period and for each day that has been considered by an optimization model based on traffic link counts.

3. Cluster analysis. A clustering algorithm is used for detecting mobility pattern patterns from the O-D matrices. These patterns are formally represented using Fuzzy prototypes Zadeh (1982).

4. Timing plan optimization. In this step of the procedure, the new timing plans will be computed for each mobility pattern that has been identified in the previous step following a static approach.

Now an extensive explanation of these steps is given in order to understand the off-line phase completely.

\subsubsection{Data collection}

Taking advantage of the new monitoring technologies it could be possible to calculate the volume of vehicles that are being driven at the time interval $t$ thanks to entrance/exit sensors located at each lane. Thus the number of vehicles which are detected by each sensor can be known, although their destinations are unknown owing to the turns that they may make.

For this work an intersection is considered described by $m$ entrances and $n$ exits, and the analyzed time period is divided into $N$ intervals, the so-called Time-of-Day (TOD) intervals $(t=1, \ldots, N)$. Under these conditions the input data for the estimation model would be:
- Entering flow $(q(t))$, i.e., total number of vehicles passing the entrance $i$ of the intersection in a given time interval $t$.

$$
\begin{gathered}
q_{i}(t)(i=1, \ldots, m) ; \\
q(t)=\left[q_{1}(t), \ldots, q_{m}(t)\right]^{T} ;
\end{gathered}
$$

$T$ indicates the transpose of a vector.

- Exiting flow $(y(t))$, i.e, total number of vehicles passing each exit $j$ of the intersection in a given time interval $t$.

$$
\begin{gathered}
y_{j}(t)(j=1, \ldots, n) ; \\
y(t)=\left[y_{1}(t), \ldots, y_{n}(t)\right]^{T} ;
\end{gathered}
$$

These data are the traffic link counts at the intersection during the time period $t$. Thus the number of vehicles which are detected by each sensor is obtained, however their destinations (exits of the intersection) are unknown.

\subsubsection{Estimation of the dynamic traffic $O-D$ matrices}

The estimation model shown in this section is based on the work Li and De Moor (1999) and it is used for obtaining the O-D matrix for each time interval $t$ on basis $q(t)$ and $y(t)$ (turns). Cremer and Keller (1987) and Nihan and Davis (1987) propose linear estimation models which omit the necessary time to cross the intersection however they introduce a random variable used for estimating the flow during the different TOD intervals. Bell (1991) and Sherali et al. (1997) present models based on traverse time. In our paper, we will use the following estimation model which creates an O-D matrices. These O-D matrices are the input of the cluster analysis phase.

One of the greatest advantages of using this estimation is that it can be computed instantaneously by using the information captured by the sensors. This is especially useful when implementing a real-time system using this model.

The notation used in this paper is listed in Table 1 for convenience. In order to illustrate this notation the intersection in Figure 3 is considered. The number of line are $n=m=4$, it is necessary to define the following variables in this example:

$$
\begin{aligned}
b_{1}(t) & =\left[b_{21}(t), b_{31}(t), b_{41}(t)\right]^{T} \\
b_{2}(t) & =\left[b_{12}(t), b_{32}(t), b_{42}(t)\right]^{T} \\
b_{3}(t) & =\left[b_{13}(t), b_{23}(t), b_{43}(t)\right]^{T} \\
b_{4}(t) & =\left[b_{14}(t), b_{24}(t), b_{34}(t)\right]^{T} \\
Q_{1}(t) & =\left[q_{2}(t), q_{3}(t), q_{4}(t)\right]^{T} \\
Q_{2}(t) & =\left[q_{1}(t), q_{3}(t), q_{4}(t)\right]^{T}
\end{aligned}
$$


Table 1: Notation used in the O-D matrix estimation

\section{Sets and elements}

$I_{j}$ : A set of entrances that allows user to take the exit $j .(j=1 \ldots n)$

\section{Data}

$m$ : Number of entrances at the intersection.

$n$ : Number of exits at the intersection.

$N$ : Number of TOD intervals.

$M$ : Number of necessary previous TOD intervals to estimate the current traffic conditions.

$y_{j}(t):(j=1 \ldots n)$ denotes the exit traffic count at leg $j$ during the TOD interval $t$.

$y(t)=\left[y_{1}(t), \ldots, y_{n}(t)\right]^{T}$

$q_{i}(t):(i=1 \ldots m)$ denotes the entering traffic count at leg $i$ during the TOD interval $t$.

$q(t)=\left[q_{1}(t), \ldots, q_{m}(t)\right]^{T}$

Variables

$b_{i j}(t)$ : The probability that a vehicle enters via $i$ and takes the exit $j$ at TOD interval $t$.

$\left(i=1, \ldots, m ; j=1, \ldots, n ; t=M, t_{1}, \ldots, N\right)$

$b_{j}(t)$ : The probability vector from each entrance $i$ to the exit $j$, at TOD interval $t$.

$b_{j}(t)=\left[b_{i j}(t)\right] \forall i \in I_{j}$;

$b(t): \quad$ A probability vector defined by $\left(\ldots, b_{i j}(t), \ldots\right)$.

$Q_{j}(t)$ : A vector based on $q_{i}(t)$ with $i \in I_{j}, j=$ $1, \ldots, n$ and $t=1, \ldots, N . Q_{j}(t)=\left[\ldots, q_{i}(t)^{T}, \ldots\right]^{T}$ where $i \in I_{j}$ appears in an increasing order.

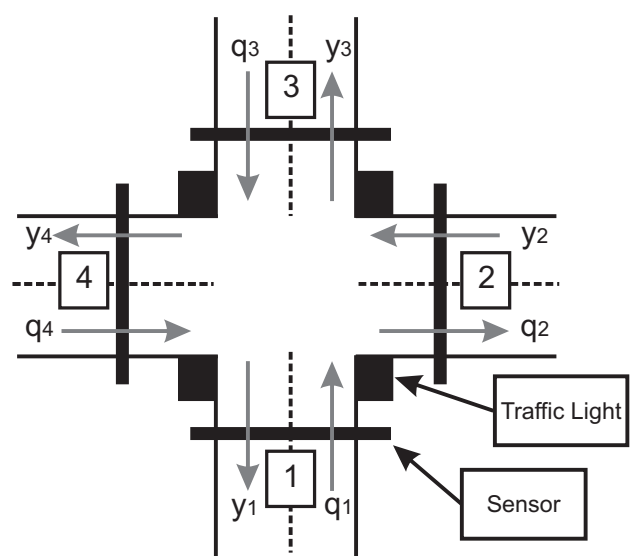

Figure 3: $q_{i}$ entrances and $y_{i}$ exits in a four lane intersection with prohibited U-turns

$$
\begin{gathered}
Q_{3}(t)=\left[q_{1}(t), q_{2}(t), q_{4}(t)\right]^{T} \\
Q_{4}(t)=\left[q_{1}(t), q_{2}(t), q_{3}(t)\right]^{T} \\
b(t)=\left[b_{21}(t), b_{31}(t), b_{41}(t), b_{12}(t), b_{32}(t), b_{42}(t),\right. \\
\left.b_{13}(t), b_{23}(t), b_{43}(t), b_{14}(t), b_{24}(t), b_{34}(t)\right]^{T}
\end{gathered}
$$

Where $b(t)$ should fulfil:

$$
b_{i j}(t) \geq 0 ; \sum_{j=1}^{n} b_{i j}(t)=1 ; b_{i i}(t)=0
$$

Therefore, every individual probability must be greater than or equal to 0 , the sum of probabilities from of entering via the fixed entrance $i$ and exiting via any $j$ must be 1 and the probability of U-turns is zero. Let $y(t)$ and $q(t)$ be the observed linkcounts for each time interval $t$. The following optimization model calculates the probability $b(t)$ at the TOD $t$ according to:

$$
\begin{aligned}
& \min Z(b(t))=\sum_{s=t-M}^{t} \sum_{j=1}^{n}\left\{y_{j}(s)-Q_{j}(s) \cdot b_{j}(t)\right\}^{2} \\
& \text { subject to }(1)
\end{aligned}
$$

where it is considered that the problem is solved for each TOD $t=M+1, \ldots, N$; and the operation $\cdot$ is the scalar product between vectors.

After resolving the previous optimization problem, demand between the origin $O_{i}$ and the destination $D_{j}$ in the TOD interval $t$ is estimated by:

$$
g_{i j}(t)=b_{i j}(t) \cdot q_{i}(t)
$$

\subsubsection{Cluster analysis}

Cluster analysis is a statistical technique that sorts observations into similar sets or groups. In this work, a clustering process is carried out in order to find relationships among the O-D matrices and to extract mobility patterns. The O-D matrices estimated by (3) are the inputs of this process. For simplicity we assume in the notation that any destination $D_{j}$ can be accessed from any origin $O_{i}$

Our clustering algorithm takes as input the similarity matrix (or graph) between the O-D matrices returned by the estimation model. The similarity between the O-D matrices is computed to be inversely proportional to the Euclidean distance (Eq. 4) between the matrices.

$$
d(v, u)=\sqrt{\sum_{i=1}^{n} \sum_{j=1}^{m}\left(v_{i j}-u_{i j}\right)^{2}}
$$

Once the similarity matrix has been computed, the clustering process is carried out following these steps: 
1. The first step is to find groups of similar O-D matrices detected in successive time intervals. This goal is achieved using a graph-based clustering method Kawaji et al. (2001).

2. After the first step, the whole O-D matrices can be well described by fuzzy graph of sub-clusters, i.e., a graph where each node represents the centroid of each sub-cluster, each edge represents the connectedness of the sub-clusters and the weight represents the fuzzy-connective-degree (i.e. distance) between two sub-clusters. Therefore, in order to detect other similar groups that exist in non-successive intervals it has been implemented a modification of the fuzzy hierarchical clustering algorithm presented in Dong et al. (2006).

A two-stage clustering, graph-based and hierarchical, has been chosen because it reflects a reasonable compromise between complexity and efficiency.

\subsubsection{Representation of mobility patterns}

The goal of this step is to represent the extracted mobility patterns. Every cluster represents a mobility pattern found at the intersection and every pattern is described by a fuzzy deformable prototype Olivas and Romero (2000) that finally will be represented by a type 1 fuzzy set.

The fuzzy sets haven been represented using triangular shape (Figure 4). For this purpose, it is necessary to obtain the centroid $\left(c_{i}\right)$ of each prototype $\left(P_{i}\right)$ and the distance from zero to the centroid of the first prototype $\left(c_{1}\right)$ which is used as the standard distance for constructing the base of the triangles (5):

$$
c_{i+1}-c_{i} \leq \Phi
$$

where $\Phi$ is the orthogonal projection of the side of the triangle over axis $\mathrm{X}$.

These parameters are obtained by a normalization and aggregation process using the O-D matrices of each cluster. The bound of each prototype is the cut with the $\mathrm{X}$ axis (coefficients $a_{i}$ and $b_{i}$ ).

Using a fuzzy numbers-based representation, it is easy to calculate the membership degree (in range [0 1]) between real traffic situations and the detected mobility patterns (prototypes) as it is shown in Section 2.2.3

\subsubsection{Timing plan optimization}

Every detected mobility pattern is defined by means of the fuzzy prototype and every prototype completes its definition with its optimal timing signal planning. This

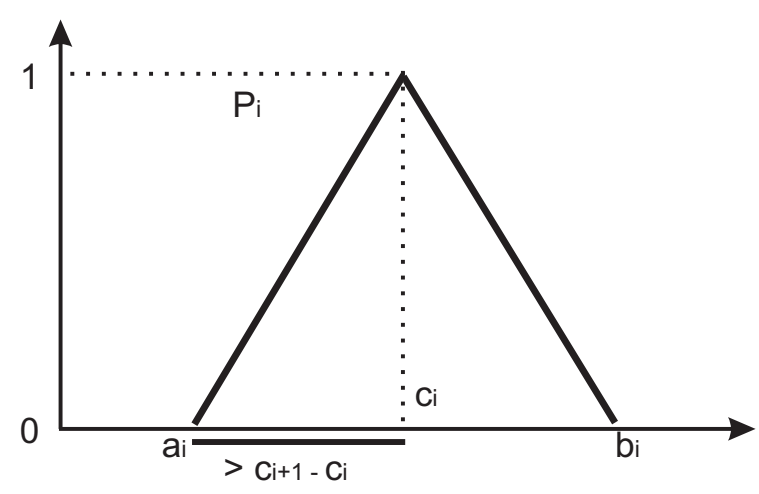

Figure 4: Fuzzy Prototype Representation

planning contains the cycle length of a signal, green splits and offsets.

The use of fuzzy deformable prototypes and the optimal timing signal planning for each prototype is a flexible solution for problems like traffic tie-ups.

\subsection{On-line stage}

Once a set of timing signal plans have been calculated, it can be applied to the real-time management of the intersection. The input data of the regulation system will be the real-time flow observations, and the exits will express the type of regulation that must take place at each moment.

The steps of this stage are pointed out now.

\subsubsection{Real-time flow observations}

At an intersection, sensors located at every entrance/exit of the lanes continuously capture information about the number of vehicles driving continuously. These data feed the system to discover the optimum traffic signal regulation parameters. This technology allows the calculation of $q_{i}(t)$ and $y_{i}(t)$ in the current TOD interval $t$, using flow data in the $M$ previous periods the current O-D matrix can be calculated.

\subsubsection{Estimation of the current $O-D$ matrix}

The estimation model described in Section 2.1.2 allows us to obtain the complete O-D matrix (including turns) from the link counts. Also the elements and calculations specified in Section 2.1.2 will be applied in this step.

\subsubsection{Inference using Fuzzy prototypes}

In this section it is shown how to calculate the most suitable mobility pattern for each situation. For this purpose, an inference process based on the fuzzy deformable prototypes of the model is used. The inputs of 


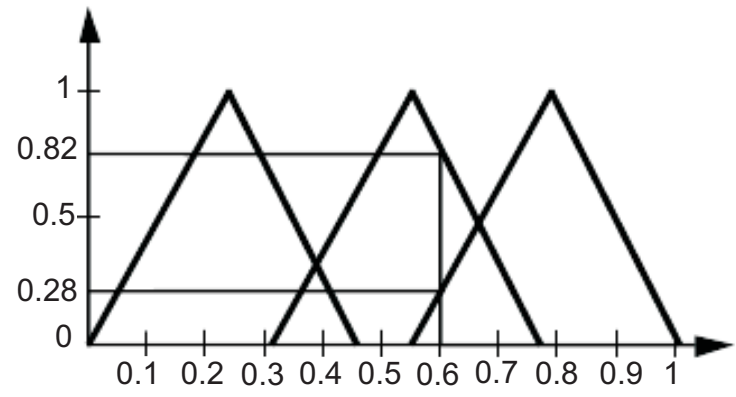

Figure 5: Similarity between a vector and a prototype $\left(X=0.6, \mu_{2}=\right.$ $\left.0,82, \mu_{3}=0,28\right)$

this process are the values of the O-D matrix previously obtained.

The process is as follows:

1. Normalization of the values of the input O-D matrix by means of the maximum and minimum coefficients of the data used in the cluster analysis. The results are values between 0 and 1 .

2. Calculate the average of the previously normalized values (this value is called $\mathrm{X}$ ).

3 . From $X$, the membership degrees $\left(\mu_{i}\right)$ for each prototype $(i)$ represented by means of the fuzzy numbers (Figure 5) are obtained as follows:

$$
\begin{aligned}
& X>c_{i}=>\mu_{i}=\frac{X-a_{i}}{c_{i}-a_{i}} \\
& X<c_{i}=>\mu_{i}=\frac{b_{i}-X}{b_{i}-c_{i}}
\end{aligned}
$$

where $c_{i}$ is the center of the prototype $i$, and the coefficients $a_{i}$ and $b_{i}$ define the bounds of each prototype $i$.

The definition of the prototype $j$ with the highest value of membership will be chosen as the most suitable traffic signal plan for the current traffic state.

$$
j=\operatorname{Arg}_{\text {maximize }_{i}}\left\{\mu_{i}\right\}
$$

\subsubsection{Implementation of the timing signal plan}

Once the most similar prototype is chosen it will be implemented the associated timing signal plan will be implemented.

Thus the output system will contain all the parameters that define the traffic lights, behaviour whilst the detected mobility pattern remains. These values will be transmitted to the electronic component in charge of transmitting orders to each of the traffic lights located at the intersection.
The methodology proposes the optimization of the timing signal plan in the off-line phase because it is a time-consuming task that can be very difficult to implement in the on-line phase. It is important to point out another fact of this methodology, the set of system responses is always known and tested a priori, and for this reason wrong or extreme responses are avoided.

\section{Computational experience}

In this section a study case is carried out in order to verify the viability of the proposed methodology. The experimental procedures and the Measure of Effectiveness (MOEs) used in this section are based on the case study scenario shown in Wong and Woon (2008).

In our experiments the "MITSIMLab" microscopic traffic simulator Yang et al. (2000) is used for evaluating the impacts of alternative traffic optimization methodologies under normal and extreme traffic conditions. The traffic simulator received as input time-dependent O-D matrices based on the hourly demand distribution shown in the Figure 6.

The objectives of the experiments carried out have been the following:

i) Study the application of the proposed methodology for a case study. This objective will be described in subsection 3.2.

ii) Compare the proposed methodology with using a non-adaptive static methodology and a nonadaptive dynamic methodology under normal traffic conditions. Section 3.3 shows the obtained results.

iii) Compare the proposed methodology using a nonadaptive static methodology and a non-adaptive dynamic methodology under unusual traffic conditions. The objective of this comparison is to demonstrate that the proposed methodology is able to be tailored to real traffic conditions in a satisfactory way. The results of this study will be described in Section 3.4.

The procedure that has been carried out is described in Section 3.1.

Data generation, clustering and fuzzy algorithms have been implemented using $\mathrm{C}++$ programming language. The tests were run on a computer with a 2.66 $\mathrm{GHz}$ Intel Pentium processor Core(TM) 2 Quad CPU Q9450 and 4 GB of RAM. 


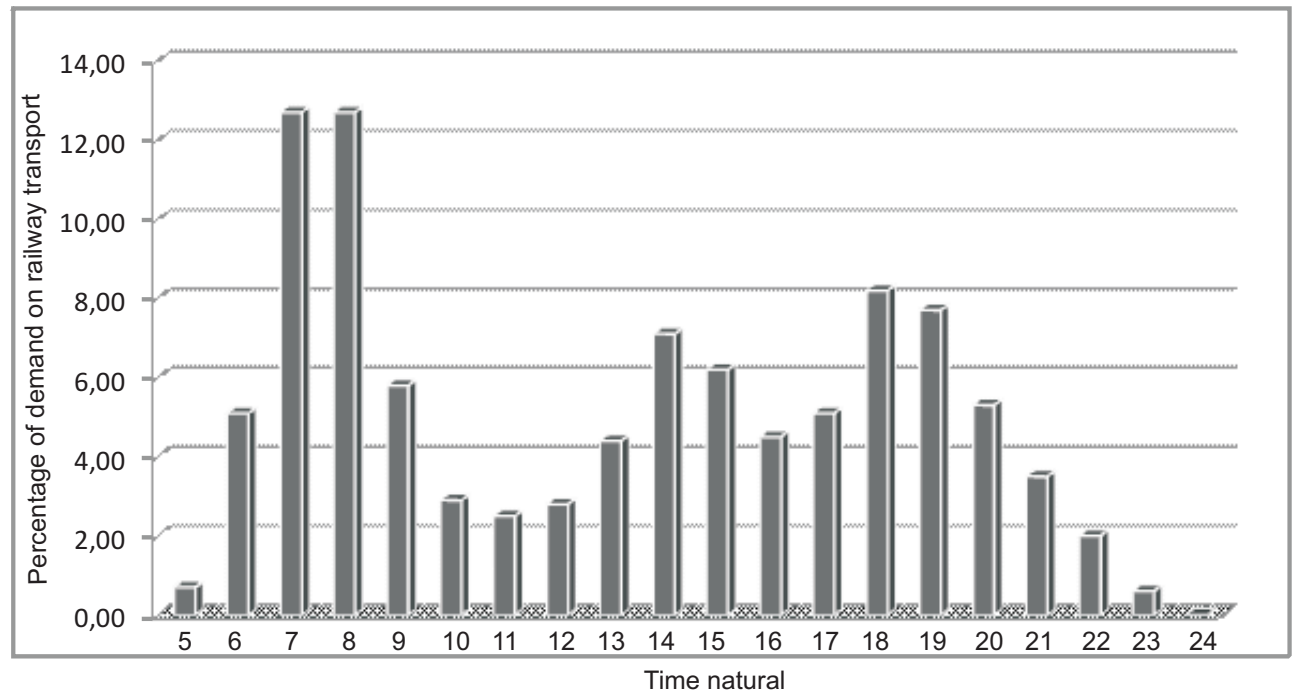

Figure 6: Hourly demand distribution $p(t)$ of the urban railway network, Madrid (Spain).

\subsection{Data generation}

An intersection as shown in Figure 3 has been considered. In this case $U$-turns are prohibited.

The data used in these numerical tests have been generated by simulation. TOD intervals are 5-minutes intervals from 5:00 am to 24:00 pm. The O-D matrix that represents the movements at the intersection has 12 columns and 228 rows. The 12 rows represent 4 entrances $x 3$ possible exits. A two-way road is supposed and the number of lanes is two per direction.

The two dynamic aspects of the demand taken into account in the production of the data are:

i) Variation of traffic flow with time.

ii) Choice of route and spatial distribution. This last point has been accounted for by time variations in the turns observed at the intersection.

Point i) is considered by the flow densities $q_{i}(t)$ and $y_{j}(t)$ which are time-dependent. Let $q_{i}(t)$ be the entering traffic density at the entrance $i$ and let $y_{i}(t)$ be the exiting traffic density at a concrete TOD interval $t$. The estimation of the entrance flows $q_{i}(t)$ in the intersection is computed by the following expression:

$$
q_{i}(t)=D * p(t) * u(1-\varepsilon, 1+\varepsilon)
$$

where:

$D$ is the total entering demand at the link, i.e., the total number of vehicles passing through the intersection throughout the day. In this simulation the fixed value 10000 vehicles has been supposed. $p(t)$ is the proportion of demand at the TOD interval $t$. Figure 6 shows the function $p(t)$ used for generating $q_{i}(t)$.

$u(a, b)$ is a uniform random variable on interval $(a, b)$. $\varepsilon$ takes the value 0.15 in ourdata.

With regard to point ii), the computation of the exits $y_{i}(t)$ at the intersection is carried out by using the expression:

$$
y_{j}(t)=\sum_{j \neq i} B_{i j}(t) * q_{i}(t)
$$

$B_{i j}(t)$ is calculated by using the expression:

$$
B_{i j}(t)=\left(\frac{t-5}{24-5}\right) * B_{1}+\left(1-\frac{t-5}{24-5}\right) * B_{2}
$$

where $B_{1}$ and $B_{2}$ are:

$$
\begin{aligned}
& B_{1}=\left(\begin{array}{cccc}
0 & 0.2 & 0.6 & 0.2 \\
0.1 & 0 & 0.5 & 0.4 \\
0.2 & 0.4 & 0 & 0.4 \\
0.25 & 0.25 & 0.5 & 0
\end{array}\right) \\
& B_{2}=\left(\begin{array}{cccc}
0 & 0.4 & 0.2 & 0.4 \\
0.5 & 0 & 0.25 & 0.25 \\
0.6 & 0.2 & 0 & 0.2 \\
0.5 & 0.4 & 0.1 & 0
\end{array}\right)
\end{aligned}
$$

After this simulation the demand O-D matrix that can be observed at the intersection and the different observed flows $q_{i}(t)$ and $y_{j}(t)$ is obtained.

Figure 7 shows the simulated data total demand $\sum_{i=1}^{N} q_{i}(t)$ for each 5-minutes TOD interval. 


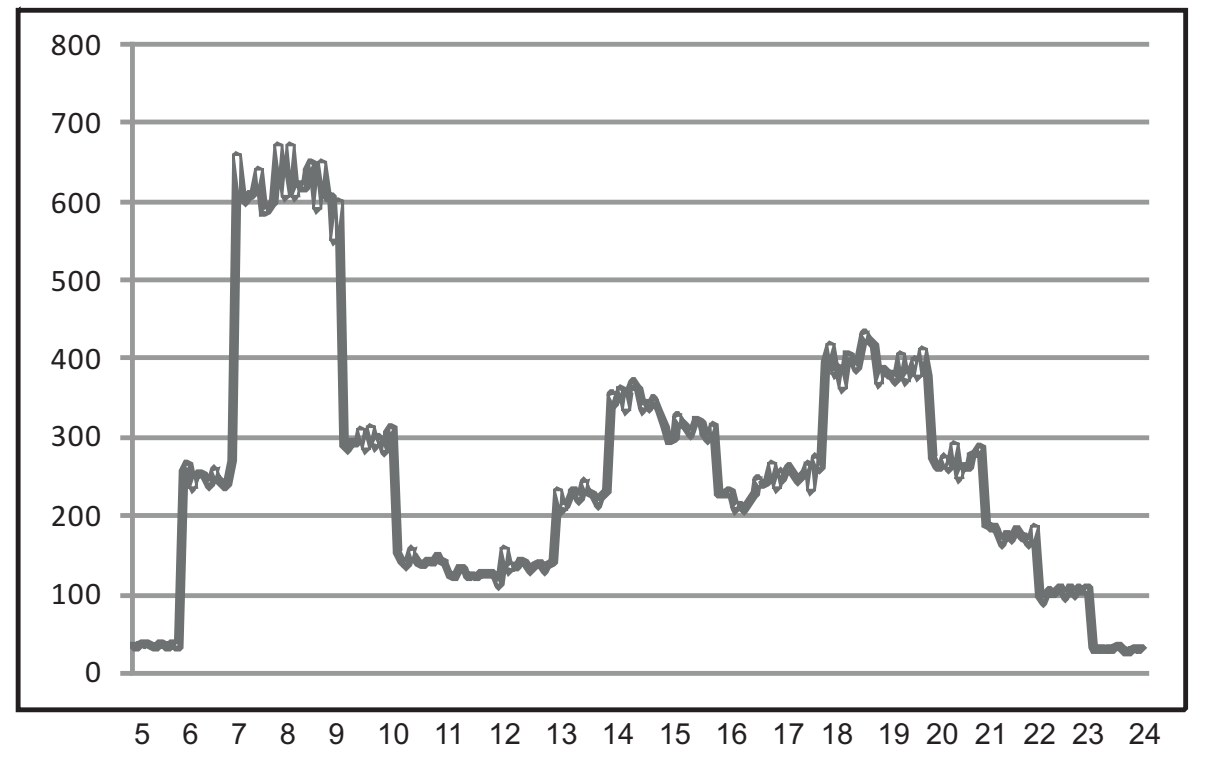

Figure 7: Simulated total demand for each TOD interval

\subsection{Results off-line phase}

This section analyzes the results obtained after employing the proposed methodology.

\subsubsection{Estimation of the traffic dynamic $O-D$ matrix}

The core of timing planning methods is the O-D matrix of the analyzed intersection. In this phase it is proposed the model described in Section 2.1.2 which estimates the O-D matrix from link counts at the intersection.

Two points are essential to the demand adjustment models: (i) the quality of the estimates and (ii) the time required to compute them. Figure 8 shows the results of the estimation model predicted demand for pair $(1,2)$ compared to the real values. These results have been computed estimating the current O-D matrix from the flows collected during the current and three previous $(M=3)$ TOD intervals because the mean relative error achieved has been $6.67 \%$. This means that the quality of the estimation model is successful.

The time used for calculating this estimation is an important parameter because it has to be computed in real time. The necessary time to process one matrix has been 0.1 seconds approximately, i.e, a reasonable time to be computed during the on-line phase of the methodology in Section 2.1.3.

\subsubsection{Clustering}

With the aim of detecting the relationships between O-D matrices and obtaining the mobility patterns, a clustering process is carried out following the algorithm previously explained.

The used clustering algorithm requires that the number of clusters $(k)$, that have to be found, has to be specified up front. Thus it is necessary to determine the optimal number of clusters, i.e., the optimal number of mobility patterns in order to obtain the best performance. For this purpose, a modification of the "elbow criterion" has been used based on the explained in Goutte et al. (1999). The process used for obtaining the optimum value of $k$ is explained as follows.

In our case, with 228 elements, the classic rule for setting the number of clusters $\left(k=\sqrt{\frac{n}{2}}\right.$, being $n$ the number of elements) computes 11 clusters as result. We have performed the clustering using a range of values for the number of clusters (from 2 to 11). Then, we have computed the within group variance of each result. The final number of clusters has been chosen in order to maximize the curvature of the within group variance. As can be seen in Figure 9 this algorithm presents results which allow us to group the elements into 5 different mobility patterns.

It is possible to consider that a higher number of prototypes is more optimum than a lesser number of them because it allows choosing a prototype closer to the current traffic situation and a better traffic signal plan. However this statement is not true, because a too high number of prototypes could cause an unstable control policy and traffic collapses.

Therefore, the O-D matrices have been grouped into 


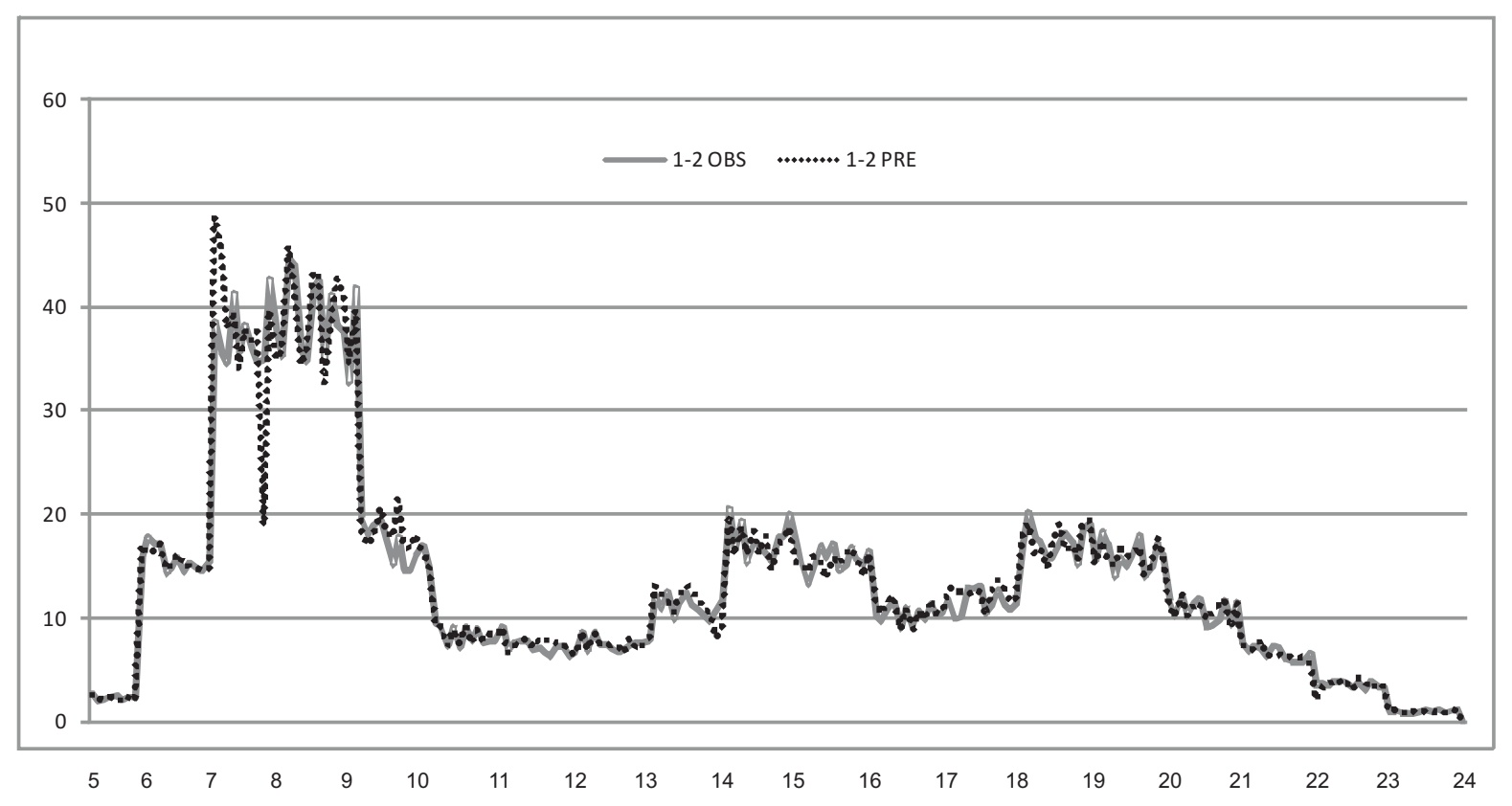

Figure 8: Observed demand pair $(1,2)$ compared to predicted demand pair $(1,2)$

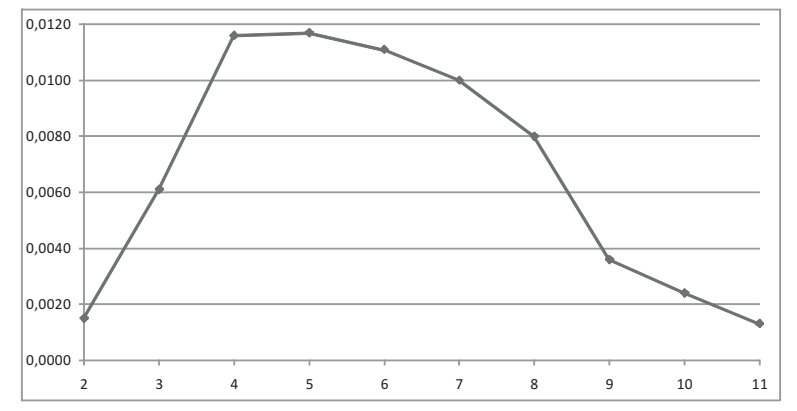

Figure 9: Variances of inter clusters

five clusters corresponding to five prototypes of traffic patterns. Considering these data prototypes and according to the values of the O-D matrices belonging to each cluster, the five prototypes were represented as fuzzy sets, which would allow us to obtain a membership degree in $[0,1]$ between a new traffic situation and each prototype. To use triangular fuzzy numbers it is only necessary to know the center of each prototype and the size of the base of the triangle (Figure 10) as was explained in Section 2.1.4.

\subsubsection{Timing plans optimization}

In this stage the optimal timing plan for each prototype discovered $P 1-P 5$ in the previous stage is defined. Nowadays there are a lot of timing optimization tools

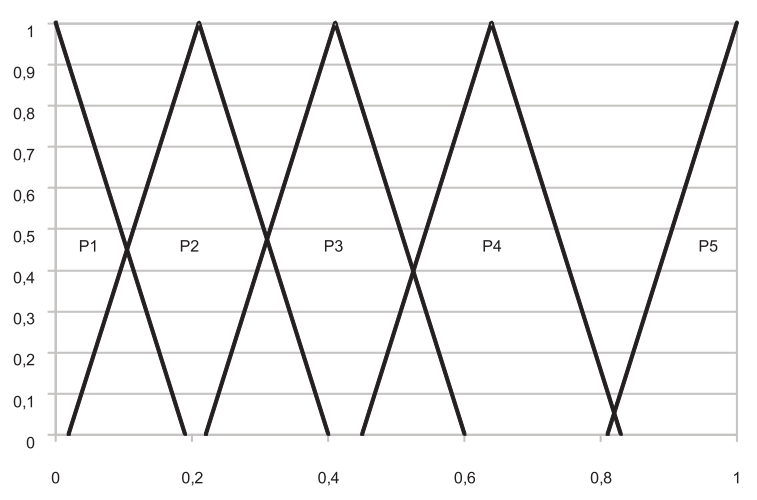

Figure 10: Prototypes of traffic patterns

such as PASSER-II Messer et al. (1974), MAXBAND Little and Kelson (1980), TRANSYT-7F Wallace et al. (1998) and SYNCHRO Trafficware (1999) which can be used in this stage. In this case the optimization for each prototype has performed by means of the trial version of PASSER V ${ }^{1}$.

An intersection has been simulated that consists of two main streets or roads and each one has two lanes per direction. One of the sides allows right turns and going straight, and the other side allows right turns and

\footnotetext{
${ }^{1}$ http://ttisoftware.tamu.edu/fraPasserV_03.htm. Last access May, 2009
} 


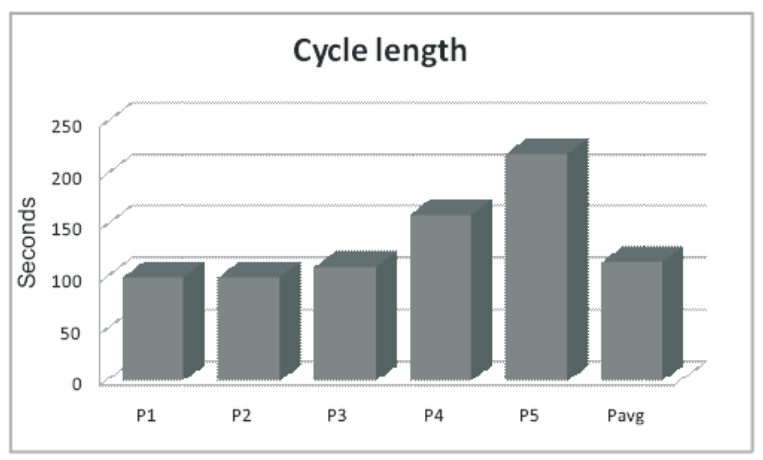

Figure 11: Cycle length obtained using PASSER V for each prototype

going straight as well. These values are the same for those prototypes which are equal.

It has been also calculated using PASSER V the optimum Traffic Light Regulation of the average pattern named Pavg, that has been calculated by the mean of the TOD intervals.

Figure 11 shows the cycle length for each prototype and the average prototype. The results obtained demonstrate that the cycle length is higher for those prototypes that represent high traffic density. Therefore the timing plans are different for each detected mobility pattern.

\subsection{Comparison between methodologies under usual traffic conditions}

The results of applying the proposed methodology, a non-adaptive static methodology and a non-adaptive dynamic methodology are compared in this subsection. For this purpose the tool MITSIMLab simulator has been used Yang et al. (2000).

The non-adaptive static methodology involves calculating the average mobility pattern and the adaptation of the timing plan for that pattern. On the other hand, the non-adaptive dynamic methodology involves the use of fixed TOD intervals Wong and Woon (2008) and the timing plan has to be adjusted using the mobility pattern of each TOD interval.

Figure 12 depicts the total sum of flow density per hour of the 12 possible movements at the intersection for each TOD interval from 5:00 am to 24:00 pm under normal traffic conditions. This graph shows the controls which use an adaptive approach and a non-adaptive dynamic approach as a function of time.

The parameters total network travel time and travel speed will be discussed in order to study the behaviour of the methodologies with respect to the simulation that has been carried out.

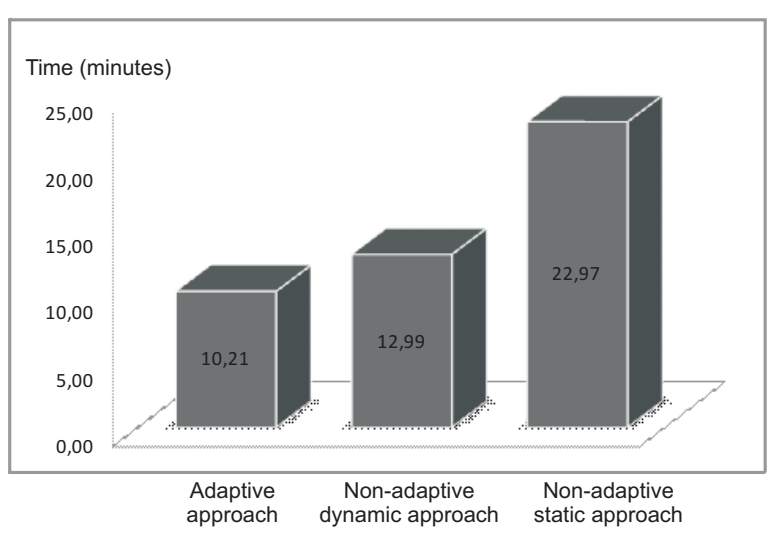

Figure 13: Total network travel times

Total network travel time: This parameter represents the average time (in minutes) that a vehicle needs to leave the intersection. To obtain a general indicator of the overall performance of the adaptive, non-adaptive dynamic and non-adaptive static approaches, first we will study the total network travel times obtained. These values can be easily obtained from the simulation and have been presented as a bar chart in Figure 13. In this case the best results have been achieved using the adaptive approach with respect to the non-adaptive dynamic approach.

On the other hand Figure 14 depicts the necessary time to pass through the intersection for each hour from 5:00 am to $24: 00 \mathrm{pm}$. Using an adaptive approach the time is reduced especially in those hours in which the traffic is denser. As can be seen in Figure 14, an inadequate timing plan can involve traffic jams and consequently it is confirmed that the timing plans have to be decided in real time according to the traffic conditions.

Travel speed: Figure 15 shows the average speeds (in $\mathrm{Km} / \mathrm{h}$ ) for each methodology and speed for each TOD interval is worse using a non-adaptive static approach and very similar using a non-adaptive dynamic approach or an adaptive approach. Figure 16 shows the speed for each methodology in each TOD interval.

Analyzing the average speed for all TOD intervals from 5:00 am to $24: 00 \mathrm{pm}$, the worst results were achieved using the non-adaptive static approach. On the other hand the results using the adaptive approach and the non-adaptive dynamic approach were very similar.

The use of the travel speed as MOE could not be really useful because it can be inconsistent depending on the circumstances. The importance of the observed difference between the travel speeds is highly dependent on the actual speed. For instance, the difference between driving at $80 \mathrm{Km} / \mathrm{h}$ and $90 \mathrm{Km} / \mathrm{h}$ is not signifi- 


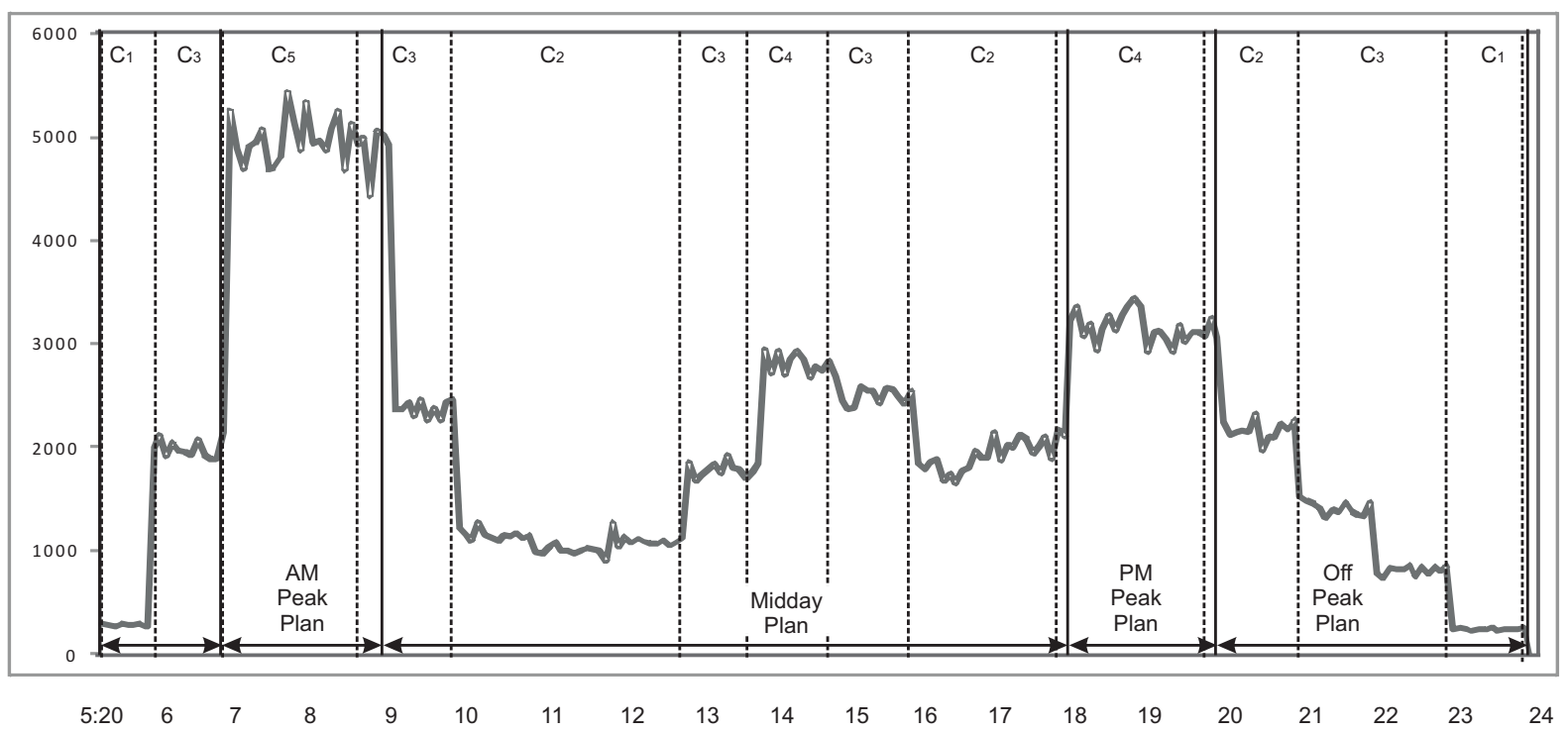

- Non-adaptive dynamic approach

------ Adaptive approach (TODs)

Figure 12: Traffic flows and controls used in the adaptive approach and non-adaptive dynamic approach as a function of time

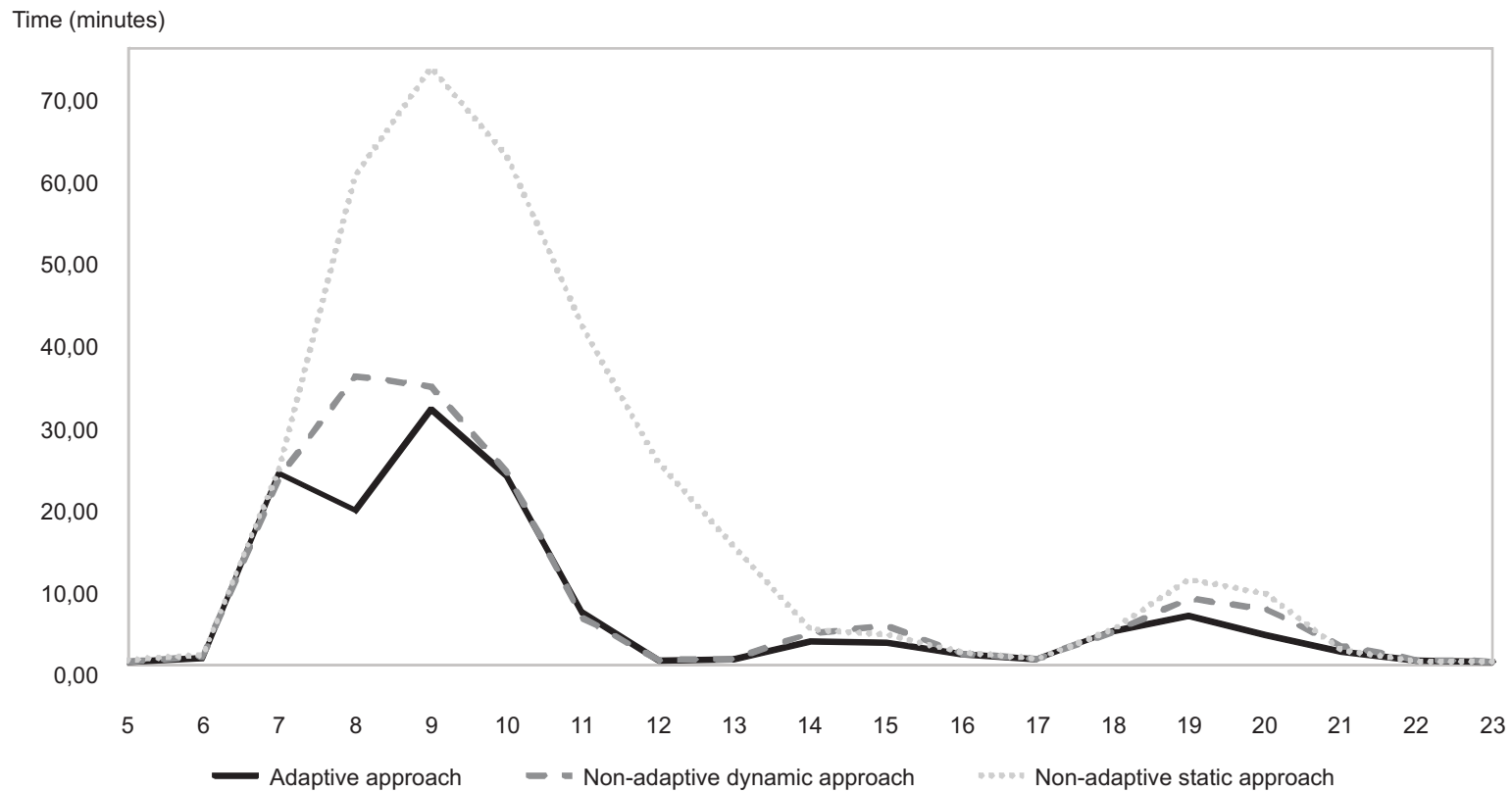

Figure 14: Travel times for each TOD intervals for methodology 


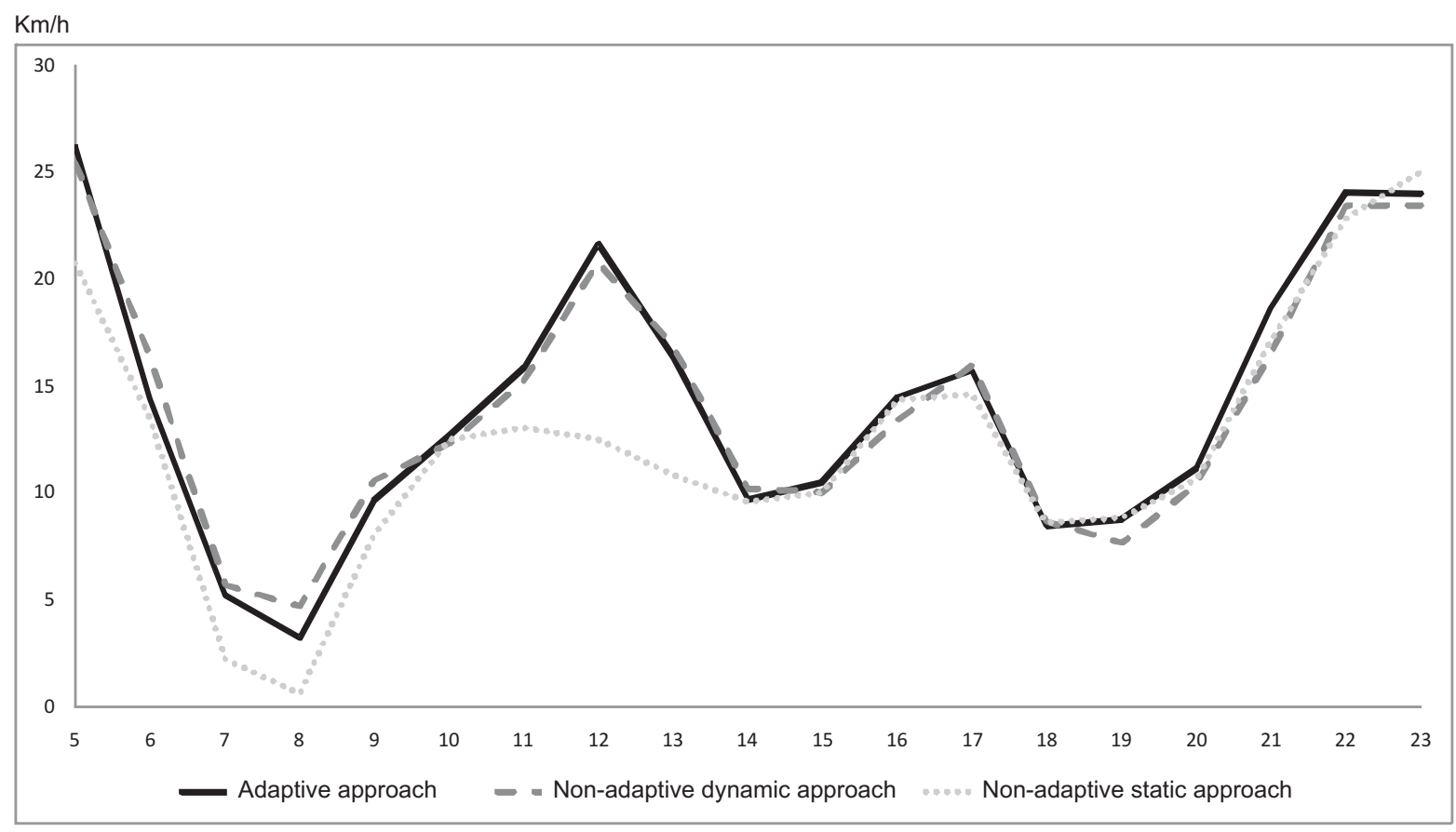

Figure 16: Travel speeds for each TOD interval and methodology

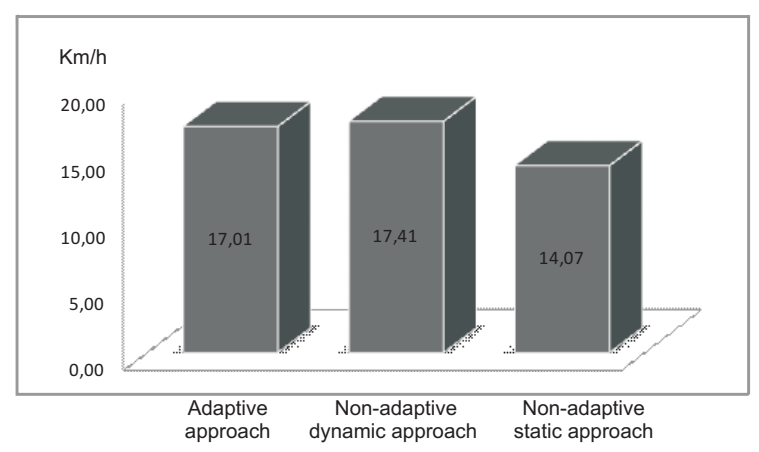

Figure 15: Average speeds for each methodology

cant but the difference between driving at $10 \mathrm{Km} / \mathrm{h}$ and $0 \mathrm{Km} / \mathrm{h}$ can be quite significant. $10 \mathrm{Km} / \mathrm{h}$ is more significant on a small lane than on a highway. In our case study an interurban intersection is supposed and consequently the small differences between speeds can be important because they can denote traffic congestion.

\subsection{Results under exceptional traffic conditions}

The results achieved by applying the three abovementioned approaches for an exceptional case. Figure 17 shows the traffic demand used for this case. This case is equal to the previous case excepting a perturbation of the demand on interval [19:00-21:00]. MITSIMLab

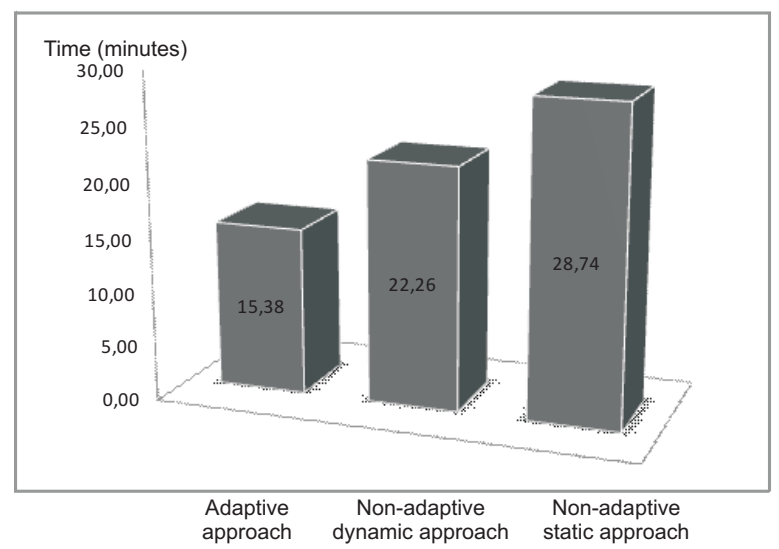

Figure 18: Total network travel times for an exceptional case

Yang et al. (2000) has been used for this comparison. In this case it will be necessary to mention again the parameters: total network travel time and travel speed.

Total network travel time: Figure 18 shows the overall performance for the three approaches. The best results have been achieved by the adaptive approach whereas the non-adaptive approaches have not been able to detect the increase of traffic flow and consequently they are not able to find the most suitable timing plan for the new traffic conditions.

Figure 19 depicts the necessary time to pass through 


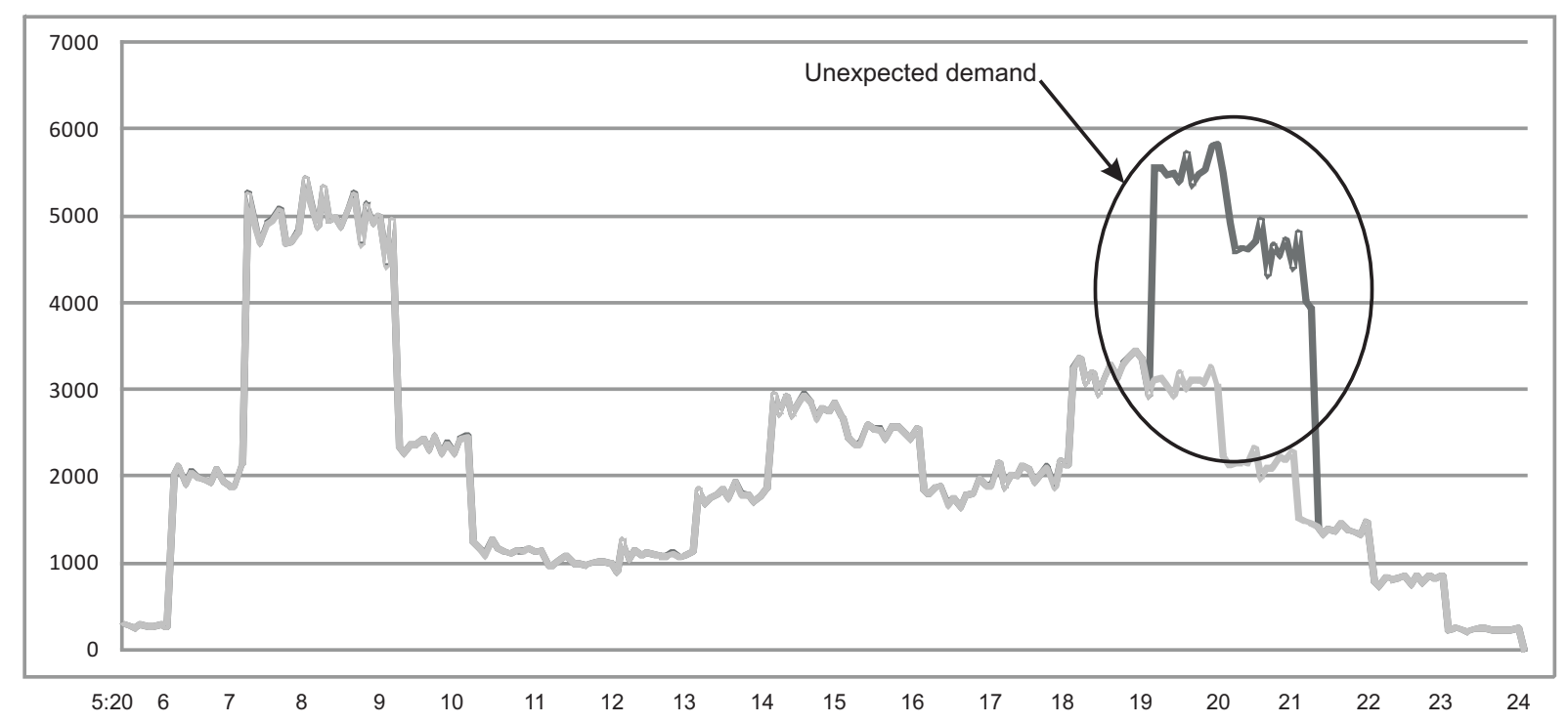

Figure 17: Traffic flow per hour at the intersection under exceptional traffic conditions

the intersection for each hour from 5:00 am to 24:00 pm. Using an adaptive approach the time is reduced especially in those hours in which the traffic is denser. As can be seen in Figure 19, an inadequate timing plan involves traffic jams and consequently it is said that the timing plans have to be decided in real time according to the current traffic conditions. Therefore, the proposed methodology obtains substantially better results for an exceptional case that increase the intensity of the flow of the traffic.

Travel speed: Figure 20 shows the travel speeds (in $\mathrm{Km} / \mathrm{h}$ ) for the the different approaches. In this case the speed for each TOD interval is worse using a nonadaptive static approach and very similar using a nonadaptive dynamic approach or an adaptive approach.

Calculating the average speed for all TOD intervals from 5:00 am to 24:00 pm, the best results were achieved using the adaptive approach improving slightly the results of the non-adaptive dynamic approach (Figure 21). On the other hand the results of these methodologies are considerably better than using the non-adaptive static approach considerably.

\section{Conclusions}

A methodology has been presented for adaptive control of traffic light intersections with monitoring technologies.

The proposed methodology is divided into two main phases, on the one hand an off-line phase and on the other an on-line phase. The off-line phase determines

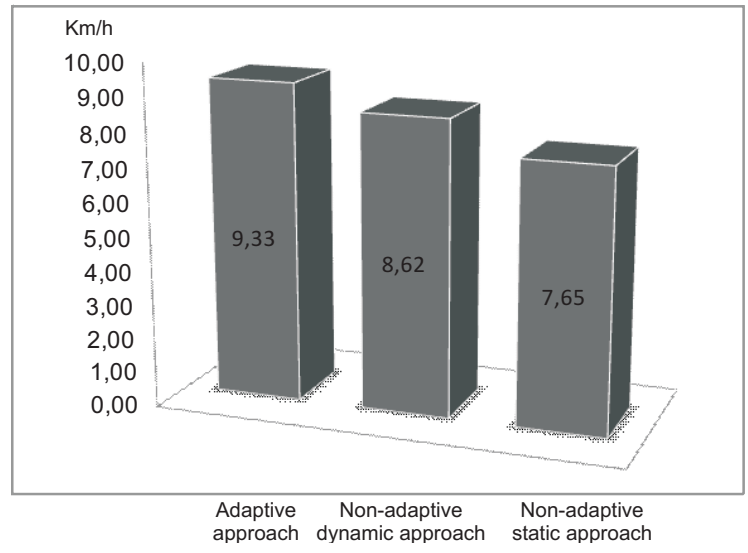

Figure 21: Average travel speeds for each methodology under exceptional traffic conditions 


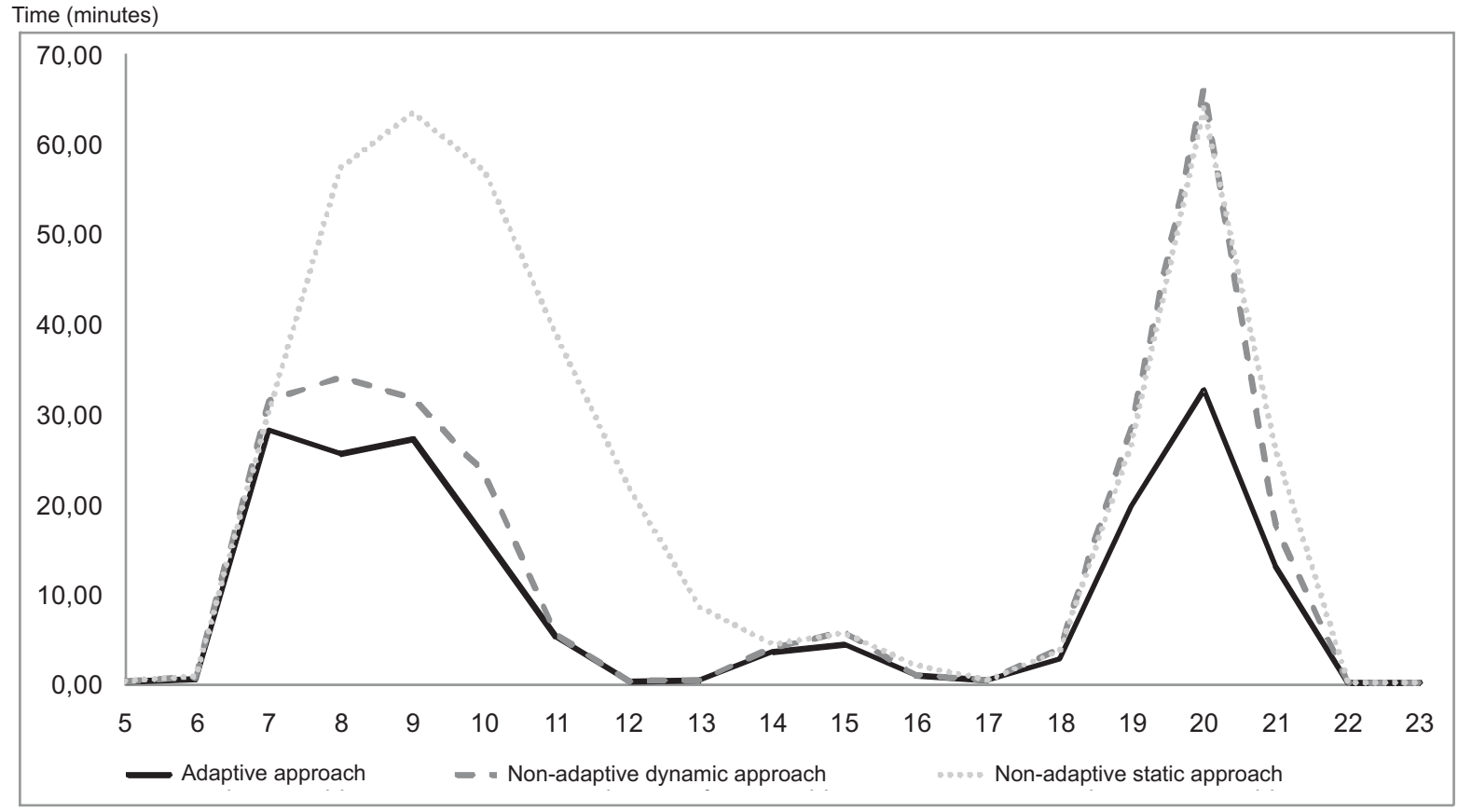

Figure 19: Travel times for each TOD interval and methodology under exceptional traffic conditions

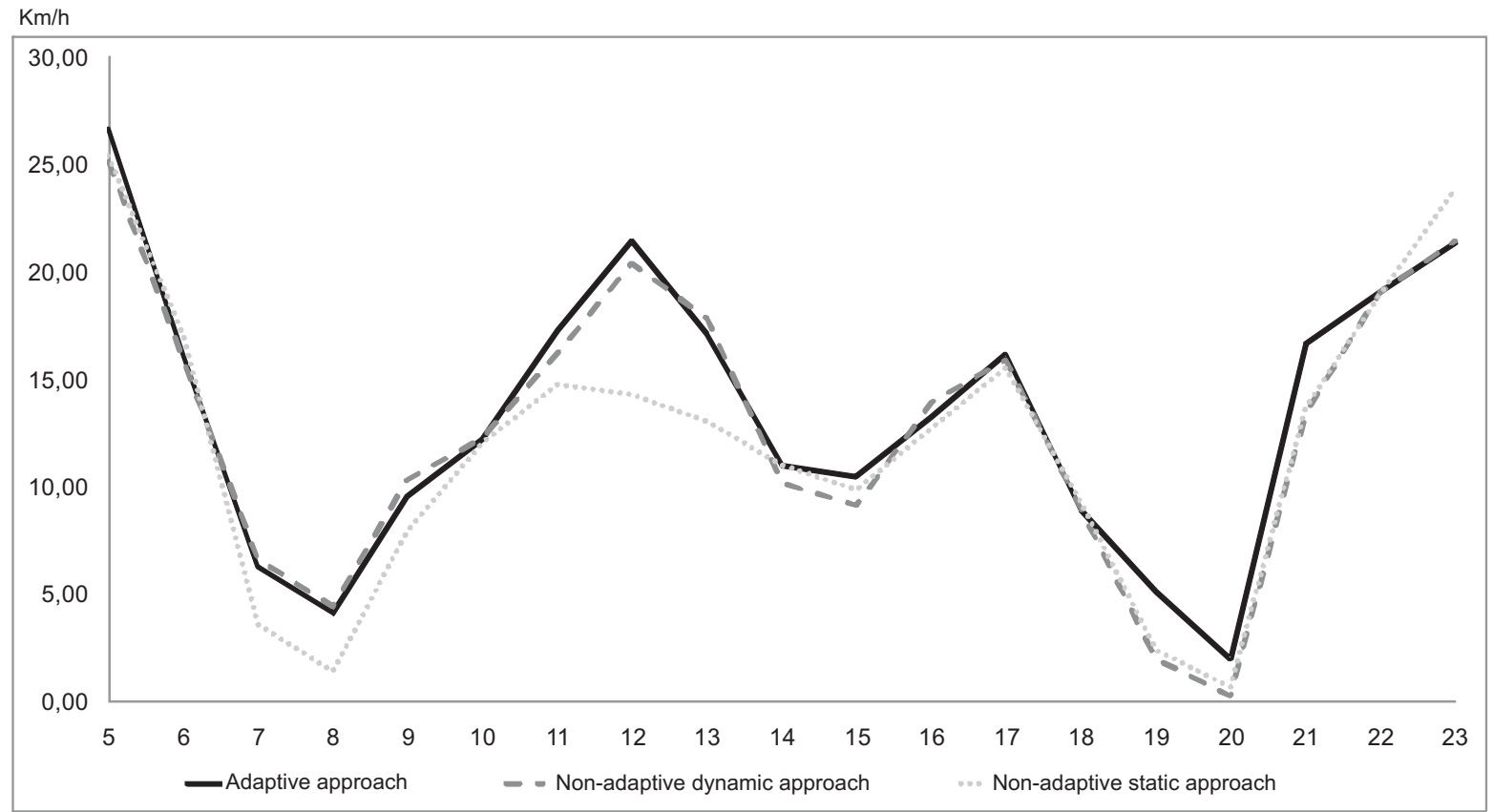

Figure 20: Travel speeds for each TOD interval and methodology under exceptional traffic conditions 
the main mobility patterns that can be detected at an intersection by means of optimization, clustering and Fuzzy techniques. After this process an optimum timing plan is decided for each mobility pattern detected in the previous phase. During the on-line phase an intelligent transportation system matches the current traffic conditions to the mobility patterns detected in the previous phase in order to identify the most suitable. This methodology means that most of the time-consuming tasks have to be carried out during the off-line phase otherwise the methodology could not work in real time.

The main advantages of the proposed methodology compared to other methodologies are: i) it is based on the most used and easily accessible data for the transportation systems such as link counts, ii) the response of the system is concrete and known a priori, this fact allows experts to avoid wrong timing plans, and iii) the on-line phase implies the execution of few simple algorithms and as a consequence the response can be computed quickly.

A case study has been implemented and tested in order to prove the proposed methodology. The results show that our methodology improves the parameters total network travel time and speed at the intersection compared to non-adaptive methodologies (static or dynamic). Also it is demonstrated that the methodology can be applied to real situations.

\section{Acknowledgments}

This research has been partially supported by PEIC09-0196-3018 SCAIWEB-2 excellence project and PCC08-0081-4388-2, of the Junta de Comunidades [Autonomous Government] of Castilla-La Mancha (Spain). TIN2007-67494 F-META project, MECFEDER, Spain.

Asar, A. U., Sadeeq Ullah, M., Ahmed, J., Riaz-ul-Hasnain, 2008. Traffic responsive signal timing plan generation based on neural network. 4th IEEE Conference on Automation Science and Engineering, CASE 2008 (4626427), 833-838.

Bell, M. G. H., 1991. The real-time estimation of origin-destination flows in the presence of platoon dispersion. Transportation Research Part B 25, 115-125.

Cremer, M., Keller, H., 1987. A new class of dynamic methods for the identification of origin-destination flows. Transportation Research Part B 21, (2), 117-132.

Dong, C., Liu, Z., \& Qiu, Z., 2005. Urban traffic signal timing optimization based on multi-layer chaos neural networks involving feedback. Lecture Notes in Computer Science 3610, (PART I), 340-344.

Dong, Y., Zhuang, Y., Chen, K., Taib, X., 2006. A hierarchical clustering algorithm based on fuzzy graph connectedness. Fuzzy Sets and Systems 157, (13), 1760-1774.

Goutte, C., Toft, P., Rostrup, E., Nielsen, F. A., Hansen, L. K., 1999. On clustering fmri time series. NeuroImage 9 (3), 298-310.
Ibrahim, A. H., Ismail, M., Jumari, K., Kiong, T. S., 2007. Development of a user-friendly application for LOS link optimization. Information Technology Journal 6, (1), 123-128.

Ibrahim, A. H., Ismail, M., Kiong, T. S., Mastan, Z. B. K., 2005. Development of software planning tools for an-intelligent traffic light wireless communication link using $5.8 \mathrm{GHz}$ WLAN. Asia-Pacific Conference on Applied Electromagnetics, APACE, Proceedings 2005, art. No. 1607847, 378-382.

Kawaji, H., Yamaguchi, Y., Matsuda, H., Hashimoto, A., 2001. A graph-based clustering method for a large set of sequences using a graph partitioning algorithm. Genome Informatics 12, 93-102.

Lee, J., Abdulhai, B., Shalaby, A., Chung, E.-H., 2005. Real-time optimization for adaptive traffic signal control using genetic algorithms. Journal of Intelligent Transportation Systems: Technology, Planning, and Operations 9, (3), 111-122.

Li, B., De Moor, B., 1999. Recursive estimation based on the equalityconstrained optimization for intersection origin-destination matrices. Transportation Research Part B: Methodological 33 B (3), 203-214.

Little, J. D. C., Kelson, M. D., 1980. Optimal signal timing for arterial signal systems, MAXBAND. Operations Research Center, MIT, Cambridge, MA, USA.

Liu, H. X., Oh, J.-S., Recker, W., 2002. Adaptive signal control system with online performance measure for a single intersection. Transportation Research Record (1811), 131-138.

López, S., Hernández, P., Hernández, A., García, M., 1999. Artificial neural networks as useful tools for the optimization of the relative offset between two consecutive sets of traffic lights. LNCS. Foundations and Tools for Neural Modeling. Berlin, Germany: Springer-Verlag, 795-804.

Ma, W.-G., Guan, H.-S., Li, A.-Z., Qin, X.-G., 2008. The research of single intersection signal control method in intelligence transportation system. Second International Conference on Innovative Computing, Information and Control, ICICIC 2007 (4427693).

Messer, C. J., Haenel, H. E., Koeppe, E. A., 1974. A report on the user's manual for progression analysis and signal system evaluation routine-PASSER II. TTI Research Report, College Station, Texas, Report No. FHWA/TX-74/165-14.

Nihan, N. L., Davis, G. A., 1987. Recursive estimation of origindestination matrices from input/output counts. Transportation Research Part B, 21, (2), 149-163.

Olivas, J., Romero, F., 2000. FPKD. Fuzzy prototypical knowledge discovery. Application to forest fire prediction. Proceedings of the SEKE'2000, Knowledge Systems Institute, Chicago, Ill. USA 24, 47-54.

Park, B., Lee, D.-H., \& Yun, I., 2003. Enhancement of time of day based traffic signal control. Proceedings of the IEEE International Conference on Systems, Man and Cybernetics 4, 3619-3624.

Rouphail, N., Park, B., Sacks, J., 2000. Direct signal timing optimization: Strategy development and results. XIth Pan American Conference on Traffic and Transportation Engineering, Gramado, Brazil.

Sánchez, J., Galán, M., Rubio, E., 2004. Genetic algorithms and cellular automata: A new architecture for traffic light cycles optimization. Proceedings of the 2004 Congress on Evolutionary Computation CEC2004 2, 1668-1674.

Sánchez, J., Galán, M., Rubio, E., 2008. Applying a traffic lights evolutionary optimization technique to a real case: "Las Ramblas" area in Santa Cruz de Tenerife. IEEE Transactions on evolutionary computation 12, (1), 25-40.

Shelby, S. G., 2004. Single-intersection evaluation of real-time adaptive traffic signal control algorithms. Transportation Research Record (1867), 183-192.

Sherali, H., Arora, N., Hobeika, A. G., 1997. Parameter optimization methods for estimating dynamic origin-destination trip-tables. 
Transportation Research Part B 31, (2), 141-157.

Spall, J. C., Chin, D. C., 1994. A model-free approach to optimal signal light timing for system-wide traffic control. Proceedings of the IEEE Conference on Decision Control 2, 1868-1875.

Stoilova, K., Stoilov, T., 1998. Traffic noise and traffic light control. Transportation Research Part D: Transport and Environment 3, (6), 399-417.

Trafficware, 1999. Synchro 4.0 Userś Guide. Trafficware, Albany, CA, USA.

Van Leeuwaarden, J. S. H., 2006. Delay analysis for the fixed-cycle traffic-light queue. Transportation Science 40, (2), 189-199.

Wallace, C. E., Courage, K. G., Hadi, M. A., Gan, A. C., 1998. TRANSYT-7F User's Guide. Transportation Research Center, University of Florida, Gainesville, FL, USA.

Wong, Y. K., Woon, W. L., 2006. Improving traffic signal control system using demining. Proceedings of the MMU international symposium of information and communication technologies, Petaling Jaya, Malaysia.

Wong, Y. K., Woon, W. L., 2008. An iterative approach to enhanced traffic signal optimization. Expert Systems with Applications 34, (4), 2885-2890.

Yang, Q., Koutsopoulos, H. N., \& Ben-Akiva, M., 2000. A simulation laboratory for evaluating dynamic traffic management systems. Transportation Research Record 1710, 122-130.

Zadeh, L. A., 1982. A note on prototype theory and fuzzy sets. Cognition 12, (3), 291-297. 